\title{
Optimization of Two-Dimensional Plasmonic Absorbers Based on a Metamaterial and Cylindrical Cavity Model Approach for High-Responsivity Wavelength-Selective Uncooled Infrared Sensors
}

\author{
Kyohei Masuda, Shinpei Ogawa ${ }^{1,2, *}$, \\ Yousuke Takagawa and Masafumi Kimata** \\ College of Science and Engineering, Ritsumeikan University, \\ 1-1-1 Noji-higashi, Kusatsu, Shiga 525-8577, Japan \\ 'Advanced Technology R\&D Center, Mitsubishi Electric Corporation, \\ 8-1-1 Tsukaguchi-Honmachi, Amagasaki, Hyogo 661-8661, Japan \\ ${ }^{2}$ NMEMS Technology Research Organization, \\ MBR99 Bldg. 6F, 67 Kanda Sakumagashi, Chiyoda-ku, Tokyo 101-0026, Japan
}

(Received December 17, 2013; accepted February 26, 2014)

Key words: uncooled infrared sensor, wavelength selective, plasmonics, cylindrical cavity resonator, metamaterial

The absorption characteristics of a two-dimensional plasmonic absorber (2D-PLA) were investigated to realize a high-performance uncooled infrared (IR) sensor. Theoretical calculations and sensor measurements indicate a threshold for the filling factor of the ratio of the dimple diameter to the period of the 2D-PLA to obtain sufficient absorption over $80 \%$. Two theoretical approaches were used to determine the absorption threshold: the plasmonic metamaterial approach and the cylindrical cavity model. The mode analysis of the cylindrical cavity resonator demonstrated that the minimum resonant mode gives a good explanation of the absorption threshold, and the filling factor of over $60 \%$ confirms that sufficient absorption can be achieved. The strong absorption occurs due to the hybridization of the spoof surface plasmon mode and cavity resonance by periodic dimples. The results obtained here provide an important and general design principle for the realization of high-responsivity uncooled IR sensors in the wideband IR wavelength region.

"Corresponding author: e-mail: Ogawa.Shimpei@eb.MitsubishiElectric.co.jp

**Corresponding author: e-mail: kimata@se.ritsumei.ac.jp 


\section{Introduction}

There has been increasing interest in the advanced functions of the uncooled infrared (IR) sensor to expand its applications. ${ }^{(1,2)}$ In particular, a wavelength-selective function enables uncooled IR sensors to identify objects through their radiation spectrum, ${ }^{(3)}$ which is useful for fire detection and analytical applications such as gas analysis. Ultimately, multicolor imaging is expected to be realized by integrating different pixels with different detection wavelengths.

Recently, we have reported a wavelength-selective uncooled IR sensor that employs plasmonics ${ }^{(4-6)}$ and functions over a wide range of the middle-wavelength IR (MWIR) and long-wavelength IR (LWIR). ${ }^{(7,8)}$ Wavelength selectivity has been realized simply by controlling the surface structure of a two-dimensional plasmonic absorber (2D-PLA) and, therefore, the spoof surface plasmon polaritons (SPPs) ${ }^{(9-11)}$ without the need for external optical filters, ${ }^{(12,13)}$ mirrors, ${ }^{(14,15)}$ or multilayer structures. ${ }^{(16)}$ Square and triangular surface lattice structures of 2D-PLAs have also been investigated. ${ }^{(17)}$ In addition, the detection wavelength can be controlled according to the reciprocal lattice vector of the 2D-PLA ${ }^{(18)}$ however, the optimal surface structure of the 2D-PLA has not yet been reported. Therefore, it is important to optimize the surface structure of 2D-PLAs to achieve high absorption, which will lead to high-performance uncooled IR sensors.

In this study, the surface structure was investigated both theoretically and experimentally with the aim of obtaining high absorption. The factors determining the absorption properties of 2D-PLA are confirmed using a metamaterial and a cylindrical cavity mode approach.

\section{Simulation and Experiment}

\subsection{Simulation}

Figure 1 shows a schematic of a thermopile with the Au-based 2D-PLA used in this study. A thermopile converts thermal energy to electrical energy using thermocouples by the Seebeck effect. The temperature difference between the substrate (cold junction)

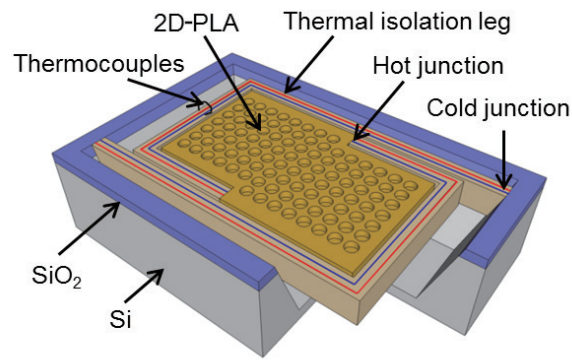

Fig. 1. (Color online) Schematic diagram of a thermopile with Au-based 2D-PLA. 
and the absorber (hot junction) is converted to a voltage, through which the IR light can be detected. The lattice structure of the 2D-PLA is square with round-shaped dimples, where the absorption wavelength is almost equal to the surface period. The effect of the dimple depth on absorption is negligible. ${ }^{(7,8)}$ Therefore, optimization of the 2D-PLA surface structure can be made only by adjusting the ratio of the dimple diameter to the fixed period at a fixed depth. The filling factor is defined as the ratio between the diameter and the period, and has a linear effect on the absorption properties in the MWIR and LWIR ranges owing to the dispersion relation with the spoof SPPs.

The absorption characteristics of the 2D-PLA were investigated. First, the absorption was calculated using a rigorous coupled wave analysis (RCWA). The diameter was varied at $3,4,5,6$, and $7 \mu \mathrm{m}$ for a fixed period of $8 \mu \mathrm{m}$ at a wavelength of $8 \mu \mathrm{m}$. The diameter was also varied at $3,4,5,6,7$, and $8 \mu \mathrm{m}$ for a fixed period of $9 \mu \mathrm{m}$ at a wavelength of $9 \mu \mathrm{m}$. The calculation results plotted in Fig. 2(a) indicate a significant increase in the absorption at the diameter threshold at around the filling factor of $50 \%$.

\subsection{Sensor fabrication and measurement}

Thermopiles with 2D-PLAs of various filling factors were fabricated through a standard complementary metal-oxide semiconductor (CMOS) method and a surface micromachining technique, as previously reported. ${ }^{(7,8)}$ The $2 \mathrm{D}$ periodical concave structure was formed on the $\mathrm{SiO}_{2}$ of the absorber area using reactive ion etching. $\mathrm{Cr} /$ $\mathrm{Au}(50 / 250 \mathrm{~nm})$ was then sputtered, where $\mathrm{Cr}$ acts as the adhesion layer between $\mathrm{Au}$ and $\mathrm{SiO}_{2}$. Sensors were fabricated in order to investigate the threshold as discussed above. The diameters for the two types of period were determined in order to cover the filling factor of approximately $40-70 \%$. The diameters were chosen as $3,4,5$, and $6 \mu \mathrm{m}$ in a fixed period of $8 \mu \mathrm{m}$ and at a fixed depth of $1.5 \mu \mathrm{m}$, and the diameters were chosen as 3,5 , and $6 \mu \mathrm{m}$ in a fixed period of $9 \mu \mathrm{m}$ and at a fixed depth of $1.5 \mu \mathrm{m}$.

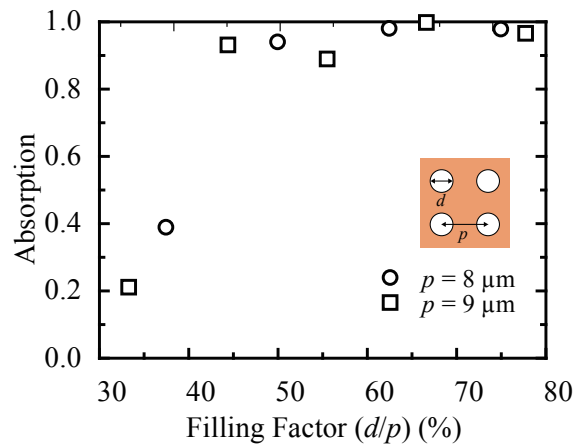

(a)

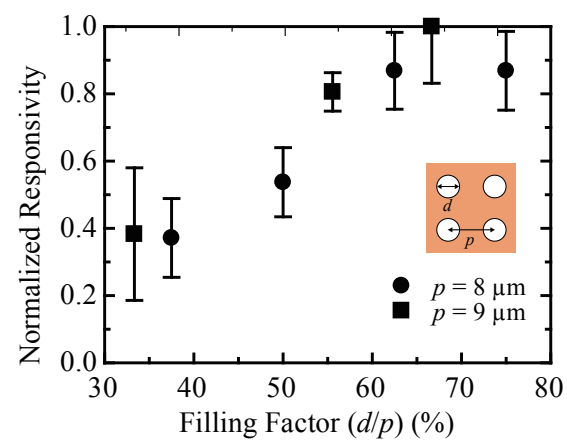

(b)

Fig. 2. (Color online) (a) Calculated absorption as a function of filling factor. (b) Normalized responsivity as a function of filling factor. 
Spectral responsivities were measured with the sensors set in a vacuum chamber with a Ge window under a pressure of $1 \mathrm{~Pa}$ to prevent thermal conduction loss through the atmosphere. The sample was irradiated with IR rays from a blackbody through narrow band-pass filters for the selection of evaluation wavelengths. The output voltage was measured and the responsivity was calculated as the ratio between the output voltage difference of the on and off states of the input power. The input power was calculated according to the measurement system parameters, absorber area, transmittance from the blackbody to the sensor through the atmosphere, narrow band-pass filters and the Ge window, and the spectral radiant emittance equation at the evaluated wavelength, as previously reported. ${ }^{(7)}$ The measured responsivities at peak wavelength are also plotted in Fig. 2(b).

The trends of the theoretical and experimental results are in good agreement. The difference between the results is attributed to structural fluctuations, such as the surface roughness and the gradient of the dimple side walls. However, Fig. 2 shows that the absorption of the 2D-PLA is not increased gradually, but is significantly enhanced beyond the threshold that exists between the filling factors of 50 and $60 \%$. When the filling factor is enlarged over $60 \%$, high absorption over $80 \%$ is achieved, which provides sufficient absorbance compared with that for typical uncooled IR sensors. Therefore, the threshold is not the local maximum value of the filling factor for the 2D-PLA to obtain sufficient absorption. The absorption properties are the advantage of the 2D-PLA owing to robustness against structural fluctuations over a wide design range of IR wavelength.

\section{Theoretical Investigation}

The threshold for the filling factor was theoretically investigated using two models.

\subsection{Plasmonic metamaterial model}

The first approach is a plasmonic metamaterial model using an effective medium approximation. Figure 3 shows that the plasmonic metamaterial approach considers the concave surface as the single effective medium, ${ }^{(9-11)}$ and the dispersion relation is calculated from the spoof plasmonic material. For simplicity, we assumed the squareshaped dimples and only take into account the specular reflected waves in order to
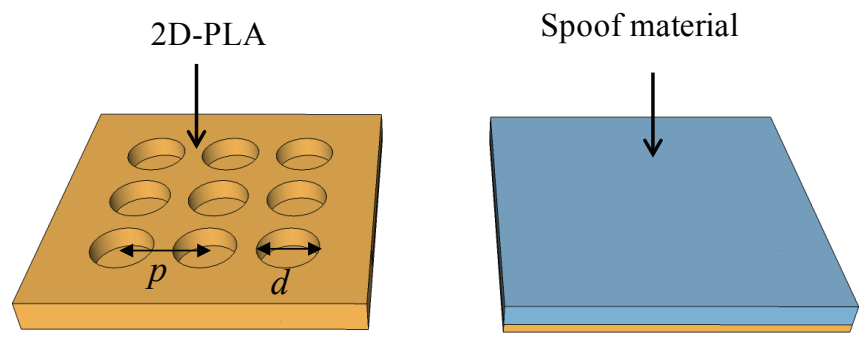

Fig. 3. (Color online) Schematic diagram of metamaterial model. 
investigate the influence of the spoof SPPs. The dispersion relation is derived from the following equations with consideration of the period, diameter, and dimple depth:(18)

$$
\begin{gathered}
\frac{\sqrt{k_{x}^{2}-k_{0}^{2}}}{k_{0}}=\frac{S^{2} k_{0}}{\sqrt{\frac{4 \pi^{2}}{d^{2}}-\varepsilon_{\mathrm{h}} k_{0}^{2}}} \cdot \frac{1-e^{-2|q| l}}{1+e^{-2|q| l}}, \\
S=\frac{\sqrt{2} d}{\pi p}, \\
k_{0}=\frac{\omega}{c_{0}} \\
q=\sqrt{\varepsilon k_{0}^{2}-\frac{4 \pi^{2}}{d^{2}}}, \\
\omega_{\mathrm{p}}=\frac{\pi c_{0}}{d \sqrt{\varepsilon_{\mathrm{h}}}},
\end{gathered}
$$

where $k_{0}$ and $k_{x}$ are the free space and surface wavenumber, $p$ is the period, $d$ is the diameter, $l$ is depth, $c_{0}$ is the speed of light in vacuum, $\varepsilon_{\mathrm{h}}$ and $\varepsilon$ are the dielectric permittivity of the vacuum and $\mathrm{Au}$, respectively, $\omega$ is the angular frequency, and $\omega_{\mathrm{p}}$ is the plasma frequency. Values of $d=3,4,5$, and $6 \mu \mathrm{m}, p=8$ and $9 \mu \mathrm{m}$, and $l=1.5 \mu \mathrm{m}$ were used for the calculation of the dispersion relation. Figure 4 shows the calculated dispersion relations. Please note that each dispersion curve is normalized by the plasma

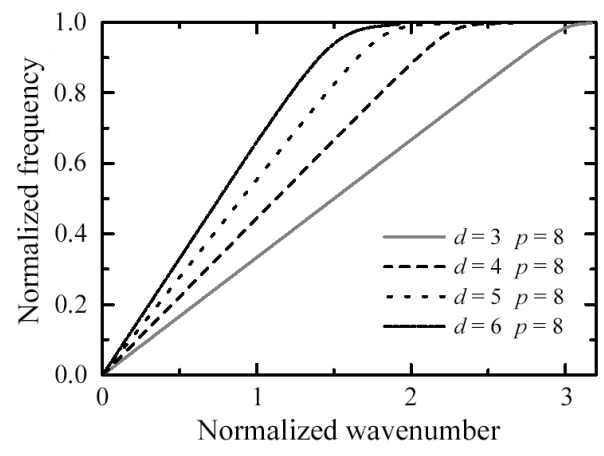

(a)

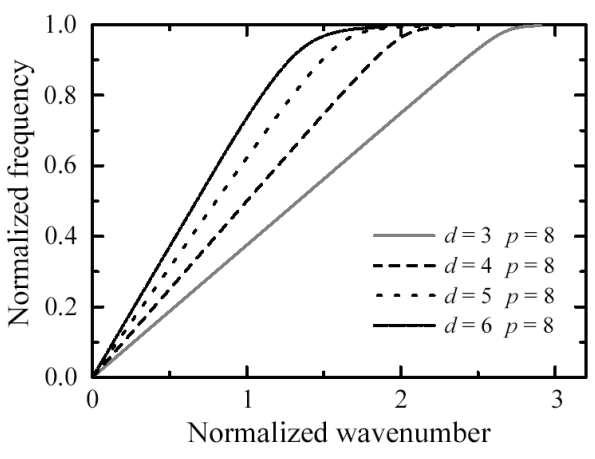

(b)

Fig. 4. Calculated dispersion relation of 2D-PLAs for various diameters and fixed periods of (a) 8 and (b) $9 \mu \mathrm{m}$. 
frequency of each $d$ for comparison. When the normalized frequency is 1 , the frequency is the plasma frequency. When the angular frequency is close to the plasma frequency, the 2D-PLA produces spoof SPP resonance, which is absorbed by the Au metal surface. Figure 4 shows that a larger filling factor causes the plasma frequency to be closer with a smaller momentum matching vector, ${ }^{(18)}$ which results in higher absorption. These results indicate that the periodic structures produce the stronger absorption according to the increase in the filling factor.

\subsection{Cylindrical cavity model}

The electromagnetic resonance inside the dimples on the 2D-PLA is the focus of the second approach. In this model, the resonance inside the dimples is considered to define the absorption of the 2D-PLA. Each dimple is considered to be a cylindrical cavity resonator. Figure 5 shows the 2D-PLA, which is considered to be a periodic array of cylindrical cavity resonators.

The absorption wavelength is the resonant wavelength of the cylindrical cavity resonator model. The resonant mode inside the dimple can be calculated using the mode theory for a cylindrical cavity resonator:(20)

$$
\begin{aligned}
& \lambda_{\mathrm{TM}}=\frac{2}{\sqrt{\left(\frac{2 u_{m n}}{\pi d}\right)^{2}+\left(\frac{s}{l}\right)^{2}}}, \\
& \lambda_{\mathrm{TE}}=\frac{2}{\sqrt{\left(\frac{2 u_{m n}^{\prime}}{\pi d}\right)^{2}+\left(\frac{s}{l}\right)^{2}}},
\end{aligned}
$$

where $\lambda_{\mathrm{TM}}$ and $\lambda_{\mathrm{TE}}$ are the resonant wavelengths for the transverse magnetic (TM) and transverse electric (TE) modes, $u_{m n}$ and $u_{m n}^{\prime}$ are the roots of the Bessel function for TM and TE mode, respectively, $d$ is the diameter of the cylindrical cavity resonator, $l$ is the depth, and $s$ is a natural number.

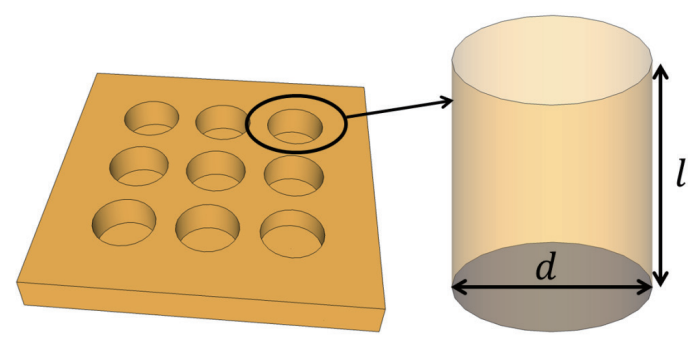

Fig. 5. (Color online) Schematic diagram of cylindrical cavity resonator model. 
The filling factor of the absorption threshold was investigated. The absorption occurs at the resonant wavelength, which is nearly equal to $p$ for the 2D-PLA. When $\lambda_{\mathrm{TM}}$ and $\lambda_{\mathrm{TE}}$ are equal to $p$, and $s$ is $0, d$ becomes minimum, which gives the filling factor of the absorption threshold. Equations (6) and (7) are thus changed to eqs. (8) and (9), respectively.

$$
\begin{aligned}
& p=\frac{\pi d}{u_{m n}} \\
& p=\frac{\pi d}{u_{m n}^{\prime}}
\end{aligned}
$$

Then, the filling factor $(d / p)$ is given by the following eqs. (10) and (11), respectively.

$$
\begin{aligned}
& \frac{d}{p}=\frac{u_{m n}}{\pi} \\
& \frac{d}{p}=\frac{u_{m n}^{\prime}}{\pi}
\end{aligned}
$$

Equations (7) and (8) indicate that the minimum of $u_{m n}$ or $u_{m n}^{\prime}$ provides the filling factor of the absorption threshold. Table 1 shows $u_{m n}$ and $u_{m n}^{\prime}$, where the $\mathrm{TE}_{11}$ mode $\left(u_{11}^{\prime}\right)$ is the minimum value.

Figure 6 shows the relationship between the value of $u_{m n}$ and the filling factor. The filling factors less than 1 should be considered as the significant parameter of 2D-PLA. There is linearity between the resonance wavelength and the diameter; when the value of the Bessel function is greater than the minimum mode $\left(\mathrm{TE}_{11}\right)$, resonance occurs. However, when the Bessel function is less than the minimum value, there is no resonance

\begin{tabular}{|c|c|c|c|c|c|c|c|}
\hline$u_{m n}$ & Value & $u_{m n}$ & Value & $\overline{u_{m n}^{\prime}}$ & Value & $u_{m n}^{\prime}$ & Value \\
\hline$\overline{u_{01}}$ & 2.405 & $u_{32}$ & 9.761 & $\overline{u_{01}^{\prime}}$ & 3.832 & $u_{32}^{\prime}$ & 8.015 \\
\hline$u_{11}$ & 3.832 & $u_{42}$ & 11.065 & $u_{11}^{\prime}$ & 1.841 & $u_{42}^{\prime}$ & 9.282 \\
\hline$u_{21}$ & 5.136 & $u_{03}$ & 8.654 & $u_{21}^{\prime}$ & 3.054 & $u_{03}^{\prime}$ & 10.173 \\
\hline$u_{31}$ & 6.38 & $u_{13}$ & 10.173 & $u_{21}^{\prime}$ & 4.201 & $u_{13}^{\prime}$ & 8.536 \\
\hline$u_{41}$ & 7.588 & $u_{23}$ & 11.62 & $u_{31}^{\prime}$ & 5.317 & $u_{23}^{\prime}$ & 9.969 \\
\hline$u_{02}$ & 5.52 & $u_{33}$ & 13.015 & $u_{41}^{\prime}$ & 7.016 & $u_{33}^{\prime}$ & 11.346 \\
\hline$u_{12}$ & 7.016 & $u_{43}$ & 14.372 & $u_{02}^{\prime}$ & 5.331 & $u_{43}^{\prime}$ & 12.682 \\
\hline$u_{22}$ & 8.417 & & & $u_{22}^{\prime}$ & 6.706 & & \\
\hline
\end{tabular}
mode in the cylindrical cavity. This minimum value is the threshold that appeared in

Table 1

Values for $u_{m n}$ and $u_{m n}^{\prime}$ for the cylindrical cavity model. 


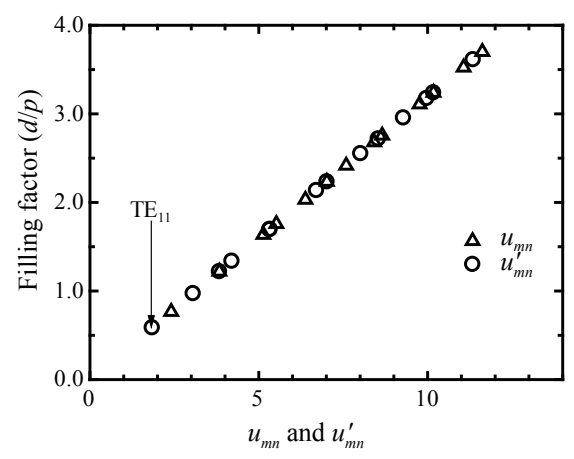

Fig. 6. Calculation results for the resonant modes of the cylindrical cavity resonator.

Fig. 2. The filling factor of the absorption threshold is defined as 0.586 ; therefore, when the filling factor is greater than the minimum value (0.586), sufficient absorption of the 2D-PLA is achieved. These experimental and theoretical results demonstrated that the filling factor is dominant to define the responsivity of wavelength-selective uncooled IR sensors with 2D-PLAs. The results of the cylindrical cavity model demonstrated that the strong absorption attributes to the cavity resonance inside the surface dimples.

\subsection{Discussion}

The first approach, i.e., the plasmonic metamaterial model, shows that the surface periodicity induces the spoof SPPs, which propagate on the metal surface and leads to the wavelength-selective absorption by 2D-PLAs. The second approach, i.e., the cylindrical cavity model, indicates that the cavity resonance inside the dimples produces the strong absorption with the cut-off filling factor. The strong wavelength-selective absorption by 2D-PLAs is attributed to the hybridization of the spoof SPPs and the cavity resonance. The hybridization of the coupling of the spoof SPPs and cavity resonance gives a good explanation to the absorption properties in Figs. 2(a) and 2(b).

\section{Conclusions}

The absorption characteristics of a 2D-PLA were investigated with the aim of realizing a high-performance uncooled IR sensor. RCWA calculations and sensor measurements indicate the threshold of the filling factor for a 2D-PLA to obtain sufficient absorption over $80 \%$. Two theoretical approaches were used to determine the threshold. The first approach was the plasmonic metamaterial approach. The spoof SPPs propagating on the metal surface cause the wavelength absorption by the metal-based periodic structure. 
The cylindrical cavity model was used as the second approach, where a dimple of the 2D-PLA is considered to be a cylindrical cavity resonator. Investigations of the minimum basic resonance mode give a good explanation of the threshold. The filling factor is over approximately $60 \%$, so that sufficient absorption can be obtained owing to the minimum resonant mode in the dimples. The absorption properties of 2D-PLAs are attributed to the hybridization of the spoof SPPs and the cavity resonance. The results obtained here provide an important design principle for the realization of highperformance uncooled IR sensors.

\section{Acknowledgements}

This work was partly supported by the New Energy and Industrial Technology Development Organization (NEDO).

\section{References}

1 M. Vollmer and K.-P. Mollmann: Infrared Thermal Imaging: Fundamentals, Research and Applications (Wiley-VCH Verlag GmbH \& Co., Germany, 2010).

2 H. Budzier and G. Gerlach: Thermal Infrared Sensors: Theory, Optimisation and Practice (Wiley, Singapore, 2011).

3 J. J. Talghader, A. S. Gawarikar and R. P. Shea: Light Sci. Appl. 1 (2012) e24.

4 C. Genet and T. W. Ebbesen: Nature 445 (2007) 39.

5 S. Kawata: Jpn. J. Appl. Phys. 52 (2012) 010001.

6 D. K. Gramotnev and S. I. Bozhevolnyi: Nat. Photonics 4 (2010) 83.

7 S. Ogawa, K. Okada, N. Fukushima and M. Kimata: Appl. Phys. Lett. 100 (2012) 021111.

8 S. Ogawa, J. Komoda, K. Masuda and M. Kimata: Opt. Eng. 52 (2012) 127104.

9 J. B. Pendry, L. Martin-Moreno and F. J. Garcia-Vidal: Science 305 (2004) 2.

10 A. Rusina, M. Durach and M. I. Stockman: Appl. Phys. A 100 (2010) 375.

11 R. Stanley: Nat. Photonics 6 (2012) 3.

12 D. Inoue, A. Miura, T. Nomura, H. Fujikawa, K. Sato, N. Ikeda, D. Tsuya, Y. Sugimoto and Y. Koide: Appl. Phys. Lett. 98 (2011) 093113.

13 Y. Ohtera and H. Yamada: IEICE Electron. Express 10 (2013) 20132001.

14 Y. Wang, B. J. Potter and J. J. Talghader: Opt. Lett. 31 (2006) 3.

15 S. W. Han, J. W. Kim, Y. S. Sohn and D. P. Neikirk: Electron. Lett. 40 (2004) 1410.

16 T. Maier and H. Brueckl: Opt. Lett. 35 (2010) 3.

17 S. Ogawa, J. Komoda, K. Masuda, Y. Takagawa and M. Kimata: Proc. Optical MEMS and Nanophotonics (OMN) (IEEE, 2013) pp. 61-62.

18 F. J. Garcia-Vidal, L. Martín-Moreno and J. B. Pendry: J. Opt. A: Pure Appl. Opt. 7 (2005) 5.

19 S.-H. Yang and P. R. Bandaru: Opt. Eng. 47 (2008) 4.

20 D. Pozar: Microwave Engineering (Wiley, New York, 1998) 2nd ed. pp. 289-290. 